\title{
Actividades de capacitación docente en Física utilizando un automóvil
}

\author{
Teacher training activities in physics using a car \\ Ernesto E Cyrulies*1,2
}

\begin{abstract}
${ }^{1}$ Centro de Investigación e Información Educativa de San Miguel, Dirección de Formación Continua, Buenos Aires, Argentina
${ }^{2}$ Universidad Nacional de General Sarmiento, Instituto del Desarrollo Humano, Los Polvorines, Buenos Aires, Argentina
\end{abstract}

Recibida en 7 de Febrero, 2017. Revisado en 25 de Marzo, 2017. Aceptado en 8 de Abril, 2017.

\begin{abstract}
Se muestran una serie de actividades realizadas en un curso de capacitación para docentes de Física, las que se desarrollaron a partir del uso de un automóvil como recurso didáctico. El curso consistió en una propuesta de trabajo cooperativo en la cual las actividades incluyeron mediciones reales tanto con el vehículo en reposo como en movimiento en diferentes situaciones. Se planteó como un ejemplo que permite contextualizar la Física, registrar datos y el posterior uso de modelos físicos. Por otro lado, las actividades propuestas requirieron la discusión sobre los métodos experimentales necesarios y sobre la validez de los modelos utilizados. Se espera que el trabajo sea inspirador para los docentes participantes, de modo que colabore al planteo de actividades más movilizadoras en la enseñanza de la Física.
\end{abstract}

Palabras clave: Capacitación docente; enseñanza de la Física; aprendizaje cooperativo.

Are shown activities of a training course for teachers of physics, which were developed from the use of a car as a teaching resource. The course consisted of a proposal of cooperative work in which the activities included actual measurements both with the vehicle at rest and in motion in different situations. It is proposed as an example that allows contextualize physics, record data and the subsequent use of physical models. On the other hand, the proposed activities have required the discussion on the necessary experimental methods and on the validity of the models used. It is expected that the work will inspire the participating teachers, collaborating with the design of interesting activities in the teaching of physics

Keywords: Teaching training; teaching of physics; cooperative learning.

\section{Introducción}

En los Centros de Investigación e Información Educativa (CIIE's), organismos dependientes de la Dirección de Formación Continua del Ministerio de Educación de la provincia de Buenos Aires, se brindan cursos de capacitación docente para diversas áreas curriculares. En dicho contexto de formación continua se brindan diferentes ofertas de capacitación en Física que promueven la reflexión sobre aspectos didácticos específicos, abordando temas que hacen a la enseñanza de la Física en el nivel secundario. Dependiendo del tipo de propuesta, en general se hace hincapié en la consideración de diversas actividades experimentales. Se asume que esta orientación promueve el desarrollo de ciertas competencias en los docentes, superando la formación inicial, particularmente en docentes noveles (los que en mayor medida asisten a estos cursos), quienes probablemente tengan sus primeros acercamientos a la construcción de dispositivos para la enseñanza, al diseño de prácticas experimentales no tradicionales, etc.

*Endereço de correspondência: ecyrulie@ungs.edu.ar
El trabajo presentado formó parte de una propuesta enmar cada en el curso "Actividades experimentales en la escuela secundaria: resignificación del trabajo de laboratorio" en uno de los CIIE's de la provincia mencionada ${ }^{1}$

El curso de capacitación incluyó actividades problematizadoras relativamente sencillas en las que se abordaron contenidos de Física; las que aquí se muestran estuvieron relacionadas con algunos aspectos técnicos de un automóvil abordables desde esta disciplina y requerían tomar mediciones o usar datos específicos para su resolución. De este modo el trabajo integró, tanto la aplicación de conocimientos teóricos en abstracto, como el trabajo sobre diseños experimentales dirigidos a completar y contrastar el conocimiento disponible, con el fin de solucionar las situaciones planteadas [1].

Según Mellado Vicente [2], desde una perspectiva más psicológica, las situaciones problemáticas con más capacidad de cambio son las que se encuentran en la zona de desarrollo próximo (ZDP) de cada profesor. Estas situaciones hacen que los profesores tengan mayor motivación y expectativas de resolverlas donde el proceso se vive

\footnotetext{
${ }^{1}$ Corresponde al CIIE de la ciudad de San Miguel, distrito de la provincia de Buenos Aires.
} 
como un desafío estimulante. Se asumió que tomar a un automóvil como caso de estudio fue generador de aquel proceso considerando que la secuenciación de actividades incluyó trabajo experimental dirigido a un objeto que genera interés. El automóvil es omnipresente en la sociedad moderna, suele ser muy apreciado y, como máquina compleja, brinda numerosas posibilidades para el planteo de problemas de Física. Más aún en un vehículo moderno, debido al mayor desarrollo de ingeniería que implica.

La dinámica de trabajo propuesta consistió en la presentación de las diferentes consignas, distribuidas en algunos de los encuentros del curso para ser discutida en grupos de trabajo cooperativos, donde las estrategias puestas en juego se construyan de modo colectivo. Entendemos que con dichos procesos se adquieren competencias desde los saberes de los cursantes y sus habilidades. En relación a esto, García Carmona [3] sostiene que el socioconstructivismo (Vigotsky, [4]), efectivamente promueve el aprendizaje de competencias. Se construye conocimiento en un marco específico, se desarrolla un proceso social de interacción y compromiso y se favorece un clima de cooperación. Según Lara [5], en una situación de enseñanza donde se organiza el aula de forma cooperativa, se permite que los participantes se ayuden entre sí y se pierde la rivalidad que habría en una organización competitiva. En ausencia de fuertes restricciones personales, las prácticas de los docentes pueden evolucionar con la condición de que esta evolución sea construida colectivamente en un trabajo cooperativo [6]. El aprendizaje cooperativo es un método que beneficia a cada uno de los participantes de la tarea; se les da la oportunidad de tener un rol más activo para enriquecer sus experiencias en el aula, pero, por otro lado, no podemos dejar de lado la intervención del docente con un rol esencial en la organización de los aprendizajes [7]. En este contexto sociocontructivista, el autor, capacitador del curso, interactuando con los asistentes tuvo a su cargo la orientación necesaria para que puedan desarrollarse según lo previsto. Las consignas que aquí se presentan, en algunos casos contenían preguntas para abrir el debate, pero también tenían como propósito considerar particularmente la resolución esperada (de este modo podría resolverse con el material y los dispositivos preparado para esa etapa del curso). Se sugirió a los docentes considerar la estrategia de resolución de problemas recomendada por Young y Freedman [8], que consiste en cuatro pasos que resumimos:

- Identificar los conceptos relevantes identificando las ideas de la física son relevantes en el problema. Aunque no se realicen cálculos, es importante para evitar resoluciones erróneas.

- Plantear el problema, determinando las ecuaciones a utilizar y dibujando, si es posible, la situación descrita.

- Ejecutar la solución haciendo los cálculos y listando las variables conocidas y desconocidas. Despejar las incógnitas.

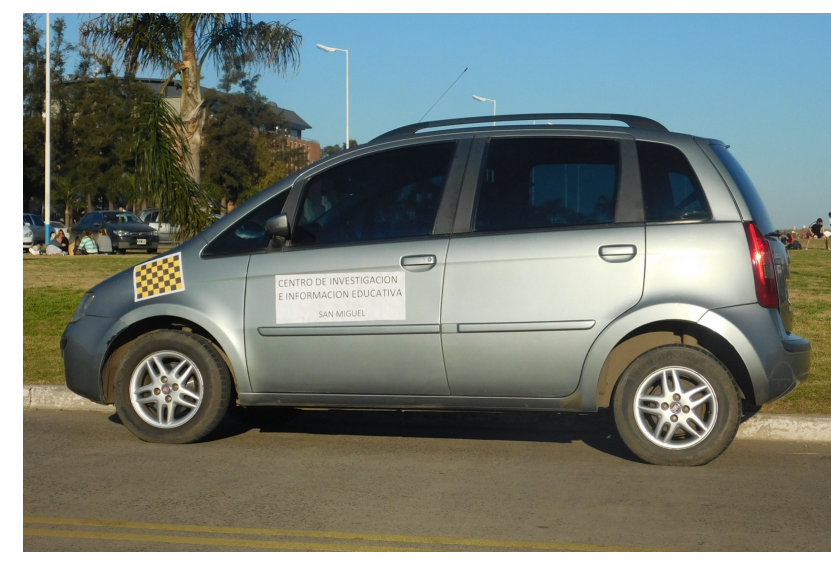

Figura 1: Vehículo utilizado para las mediciones.

- Evaluar la respuesta para considerar si es lógica, de modo que si no lo es permitirá modificar la solución.

En nuestras actividades el tercer paso conlleva además la identificación de las variables que serán medidas en la toma de datos sobre el vehículo y el modo de llevarlo a cabo.

Cabe aclarar que con la propuesta se intentó además ejemplificar sobre posibles situaciones de enseñanza fundadas en objetos cotidianos de gran utilidad potencial en términos de la didáctica disciplinar, sin esperar una transferencia directa de la propuesta al nivel secundario.

\section{Desarrollo de las actividades}

A continuación, se presentan las actividades con las consignas tal como fueron entregadas a los docentes y algunos resultados obtenidos por ellos. Por cuestiones de extensión se omiten algunas expresiones matemáticas utilizadas, aunque se describe la resolución empleada en cada caso. A los efectos de priorizar la mirada didáctica y centrar las discusiones en la resolución de los problemas y su pertinencia, no se realizó la posible propagación de errores en los cálculos vinculada a las incertezas en las mediciones. Esto no excluyó un tratamiento cualitativo de algunas de ellas. Con los mismos objetivos se ha simplificado la resolución al no considerar otras variables de menor incidencia (rendimiento de la transmisión en la caja de cambios, entre otras posibles).

Para la toma de datos hemos utilizado un vehículo marca Fiat, modelo Idea 1.4 (ver Figura 1).

Los datos técnicos ofrecidos por el fabricante que consideramos para las actividades son los que se muestran en la Figura 2 (tomados del manual de usuario).

\subsection{Actividad 1}

Para una misma marcha (o cambio), ¿será constante la aceleración a medida que aumenta la velocidad? ¿Por qué? ¿Qué método podría utilizarse para determinar la aceleración? ¿Qué datos experimentales necesitarían? ¿Podremos calcular la fuerza de rozamiento entre las 


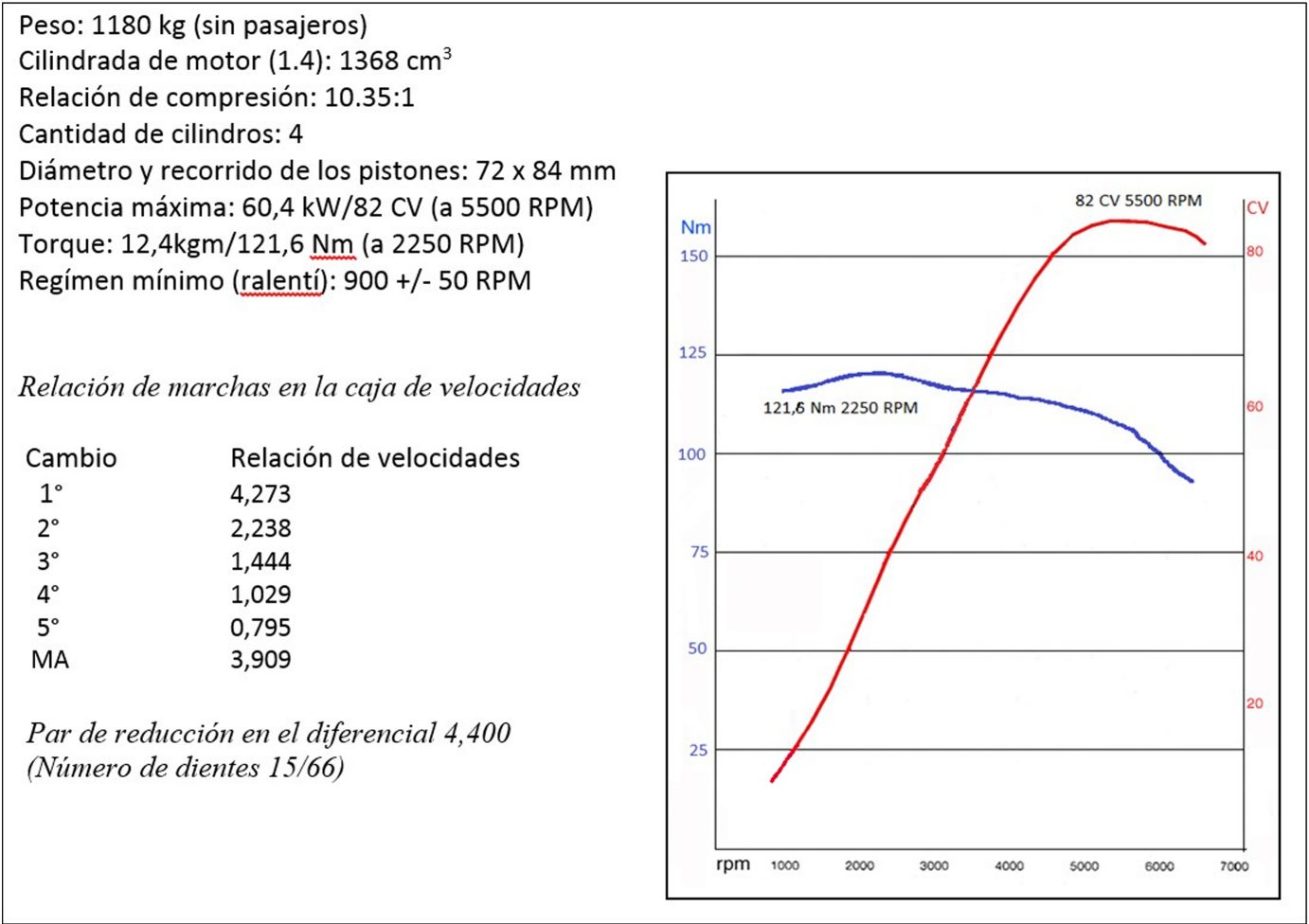

Figura 2: Datos técnicos del fabricante y curvas características del motor. Éstas corresponden al torque y potencia en función de las RPM (en relación al peso declarado del vehículo, es conveniente resaltar que suele ser un error común confundir peso con masa en la información técnica).

ruedas motrices y el piso, causante de dicha aceleración? Calcularla para la situación de máxima aceleración.

Se consideraron dos formas para la resolución. Una de ellas fue la utilización de un péndulo con un transportador de referencia en el interior del auto, libre de oscilar en el plano vertical correspondiente a la dirección de marcha en línea recta. Al mismo se lo vinculó al techo (Figura 3, arriba).

Se invitó a discutir en grupo la propuesta y a hacer las consideraciones necesarias sobre el péndulo para evaluar su uso en la determinación de la aceleración (identificando las fuerzas a la que está sometido dicho elemento haciendo el diagrama de cuerpo libre, considerándolo desde el sistema inercial y el no inercial).

En condición de marcha y a partir de su filmación se eligió una serie de fotogramas del vided ${ }^{2}$ y haciendo uso de las consideraciones dinámicas se obtuvo la aceleración (en función del tiempo).

La segunda opción acordada fue el cálculo de aceleraciones medias en pequeños intervalos de velocidad $\Delta \mathrm{v}$ filmando la evolución del velocímetro mientras se acelera el auto (Figura 3, abajo). A partir de la filmación se determinaron, eligiendo convenientemente los fotogramas en el video, intervalos $\Delta \mathrm{v}$ de $5 \mathrm{~km} / \mathrm{h}$ y sus correspon-

2 Se utilizó el programa Spanish Dub, el cual resultó muy versátil para nuestros propósitos. Es gratuito y puede obtenerse de http://spanishdub.softonic.com/. Consultado el 30 de enero de 2017 .

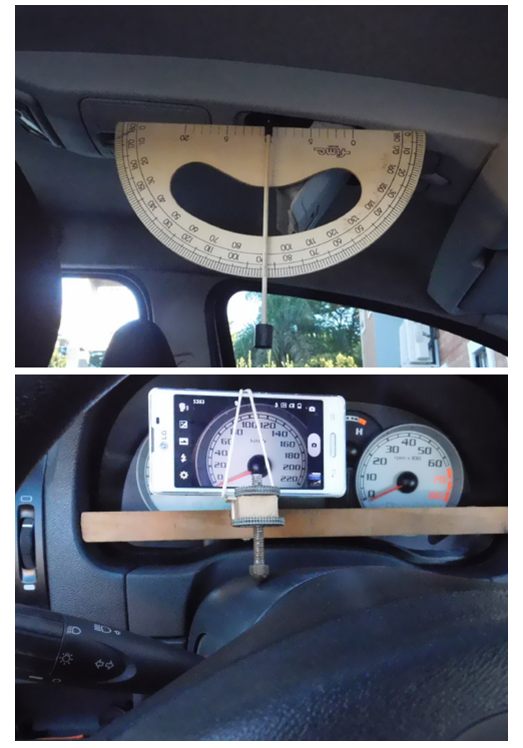

Figura 3: Arriba: Péndulo con transportador vinculado al techo. Abajo: Teléfono celular montado en dispositivo para filmar el velocímetro.

dientes $\Delta \mathrm{t}$ para el rango de velocidades considerado (de 0 a $80 \mathrm{~km} / \mathrm{h}$, límite impuesto en la ruta utilizada). Los datos obtenidos se procesaron con planilla de cálculo. Se consideraron tres marchas en la experiencia $\left(1^{\mathrm{ra}}, 2^{\mathrm{ra}} \mathrm{y}\right.$ $\left.3^{\text {ra }}\right)$. 
Puede verse en los gráficos de la Figura 4 (construidos con datos del velocímetro) que, como era de esperarse, la aceleración disminuye a medida que se seleccionan marchas más altas (con cada cambio disminuye el par en las ruedas para una misma velocidad de motor). Las interrupciones en las curvas de $\mathrm{v}(\mathrm{t})$ corresponden a los intervalos en los que el conductor cambia de marcha (esto se hace evidente además a partir de la filmación, donde se registró el sonido del motor). Se aprecia cierta convexidad en dichas curvas, lo que indicaría una leve disminución de la aceleración con la velocidad para una misma marcha. Como ejemplo de lo calculado se menciona que la aceleración media para el tramo medido en $1^{\text {ra }}$ velocidad dio como resultado $3,42 \mathrm{~m} / \mathrm{s}^{2}$. Teniendo en cuenta el dato de la masa del auto (y la de sus dos ocupantes en esa ocasión), la fuerza causante de dicha aceleración alcanzaría los $4400 \mathrm{~N}$ (calculada a partir de la segunda ley de Newton).

\subsection{Actividad 2}

¿Podemos determinar la potencia que entrega el motor a las ruedas en velocidad constante? Se propone determinarla para el caso de una velocidad de paso de hombre.

Opción: Empujar el auto a mano. ¿Podemos medir la fuerza necesaria? ¿Cómo medimos la velocidad? (es demasiado baja para que la registre el velocímetro)

$v(t)$

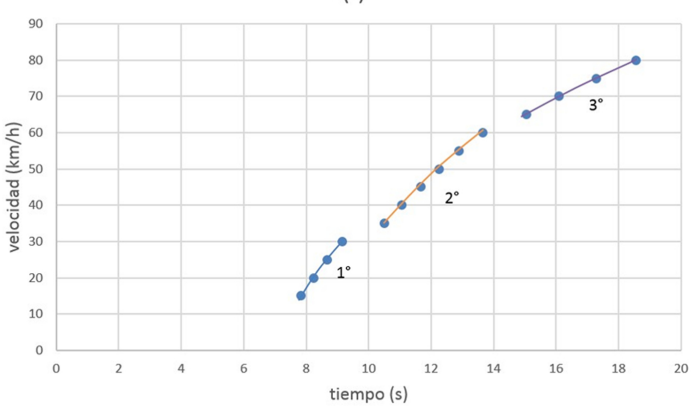

$a(t)$

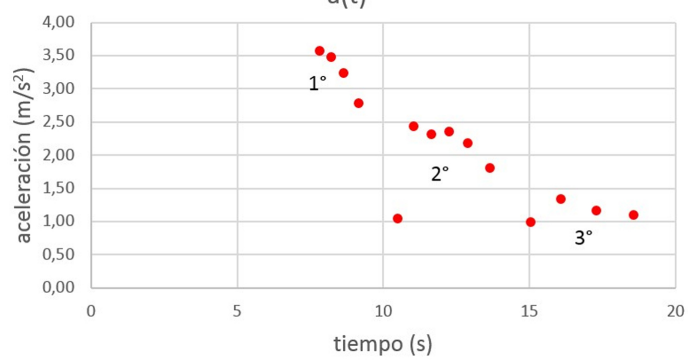

Figura 4: Gráficos construidos con los datos obtenidos en la filmación del velocímetro. Arriba: Velocidad en función del tiempo. Abajo: Aceleración en función del tiempo. Los dos puntos que indican un valor bajo $\left(1 \mathrm{~m} / \mathrm{s}^{2}\right)$ son el resultado de tomar aceleraciones medias; no representan aceleración instantánea (ninguno de los del gráfico). Los mismos se corresponden con los lapsos de cambio de marcha, los que demandan un tiempo inferior al $\Delta$ t usado para el cálculo de la aceleración.
Se empujó el vehículo manualmente por medio de una balanza hogareña apoyada en la parte trasera (Figura 5) lo que permitió medir la fuerza $F$ necesaria para mantenerlo en velocidad constante $v(230 \mathrm{~N}$ para nuestro caso, donde resultó una velocidad promedio de 4,7 $\mathrm{km} / \mathrm{h}$ ). Cabe aclarar que el valor de la fuerza proviene de una medición en la cual no resulta sencillo mantener una lectura suficientemente estable. Por esta razón, se tomó un valor promedio, luego de repetir varias veces la medición y consensuando en el grupo.

La distancia $\Delta d$ recorrida (unos $40 \mathrm{~m}$ ) fue medida con una cinta métrica y se registró el tiempo $\Delta t$ para calcular la velocidad media correspondiente.

Con estos datos empíricos se calculó la potencia necesaria que implica desplazarlo en las condiciones mencionadas (ecuación 1).

$$
P=\frac{F * \Delta d}{\Delta t}=\frac{230 N * 40 m}{30,6 s} \approx 300 W
$$

Por otro lado, con los datos de la transmisión, esto es, la relación de velocidades en caja de cambios $R C$ y la del diferencial $R D$ (Figura 2) junto al dato del radio de las ruedas $3^{3} r_{r}$ se calculó con la ecuación (2), a qué velocidad estaría girando el motor para esa situación de marcha (en $1^{\text {ra }}$ velocidad).

$$
R P M_{\text {motor }}=\frac{v * R C * R D * 1000}{2 * \pi * r_{r} * 60} \approx 858 R P M
$$

(con las constantes indicadas, $v$ se expresa en $\mathrm{km} / \mathrm{h} \mathrm{y}$ $r_{r}$ en $\left.m\right)$

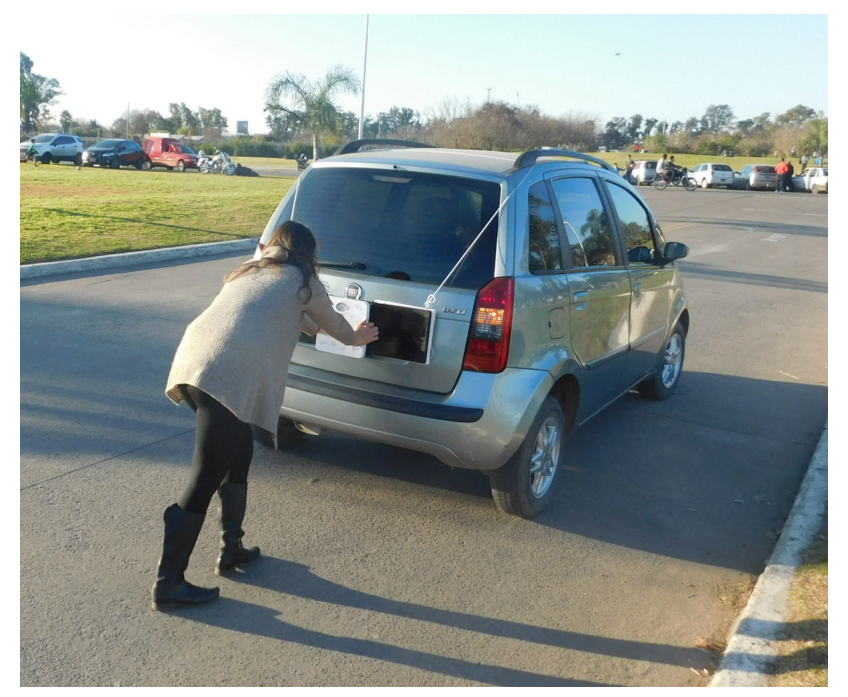

Figura 5: Profesora empujando al vehículo por medio de la balanza sobre el portón trasero en una calle horizontal solitaria. Con dicha acción se determinó la fuerza requerida para conservar el auto a velocidad constante. Dentro del vehículo permanece un conductor por cuestiones de seguridad, con el motor en marcha y las balizas encendidas.

\footnotetext{
${ }_{3}^{3}$ Estrictamente, la distancia del centro de giro al piso debido al aplastamiento parcial del neumático.
} 
Dando esto un valor que resultó dentro del rango del régimen mínimo o ralent 4 , se buscó la potencia que es capaz de entregar a esa velocidad de giro observando la curva de potencia como función de las RPM (gráfico de la Figura 2). Se calculó también a qué velocidad "debería" girar el motor en otros cambios para la misma situación de marcha. En esos casos, naturalmente, el cálculo arrojó valores inferiores a la velocidad de ralentí, por lo cual no sería posible el desplazamiento a velocidad de paso de hombre si no es en 1ra velocidad.

Resulta de esta actividad que conservar el vehículo a tan baja velocidad apenas demanda un $4 \%$ de la potencia "disponible" según lo indica la curva del motor. Este porcentaje, desde luego, aumenta en gran medida para velocidades altas del vehículo debido a la mayor presencia de fuerzas no conservativas. Cabe aclarar además que el mencionado porcentaje para velocidades mayores dependerá de la marcha utilizada, ya que para una misma velocidad de desplazamiento será diferente la velocidad de giro del motor, y con ésta también lo será la potencia entregada.

\subsection{Actividad 3}

¿Qué distancia mínima necesitaremos para frenar completamente sobre pavimento horizontal? (por ejemplo de $100 \mathrm{~km} / \mathrm{h}$ a 0 )

Contamos con algunos resortes y una balanza para las mediciones necesarias. ¿Qué método proponen para dar respuesta al problema? Discutir como intervienen ambos coeficientes de rozamiento entre los neumáticos y el piso $\left(\mu_{e}\right.$ y el $\left.\mu_{d}\right)$ en diferentes condiciones de frenado (el vehículo que utilizamos no posee $A B S$ )

Se consideró un modelo simplificado en el cual se realizaron mediciones que permitieron calcular el pestático y el $\mu$ dinámico, asociados respectivamente a un frenado normal y a otro que bloquee el giro de las ruedas 5 . Se utilizó para esto la rueda de auxilio y se improvisó un dispositivo para impedir que la misma gire mientras era arrastrada sobre el pavimento para realizar las mediciones. Las fuerzas de rozamiento (estática y dinámica) se determinaron desplazándola por medio de un resorte del cual se midió el estiramiento producido con dicha acción (Figura 6). Para completar el cálculo de la fuerza se determinó además de modo experimental la constante elástica del resorte $(K=F / \Delta x)$. El peso de la rueda (el que se tomó como equivalente a la fuerza normal $N$ ) se midió con la balanza hogareña utilizada en la actividad 2.

\footnotetext{
4 Velocidad de rotación mínima, por debajo de la cual el funcionamiento de un motor de combustión interna es ineficiente (o no es posible si es significativamente menor). Para nuestro caso corresponde a 850 RPM según se desprende de los datos del fabricante. ${ }^{5}$ La asociación referida permite un abordaje sencillo del problema. Existen estudios que dan cuenta que las condiciones dinámicas de marcha requieren considerar la variación de la adherencia con la velocidad.
}

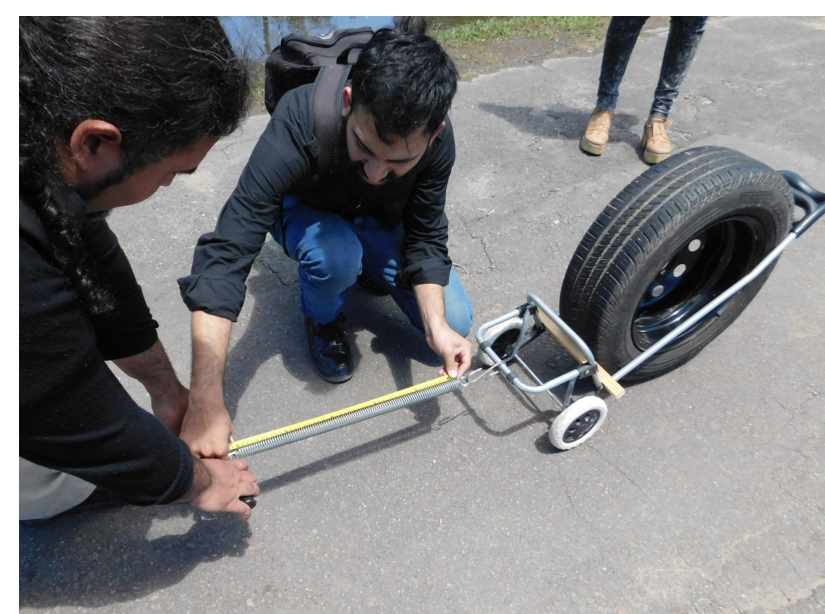

Figura 6: Medición del estiramiento del resorte utilizado para calcular la fuerza de rozamiento entre la goma y el pavimento.

La fuerza de rozamiento, según se evite o no el deslizamiento entre las superficies en contacto (goma y pavimento en la experiencia), viene dada por las ecuaciones (3) y (4):

$$
\begin{aligned}
& F r_{e} \leq \mu_{e} N \\
& F r_{d}=\mu_{d} N
\end{aligned}
$$

Con estos modelos se obtuvieron los respectivos coeficientes de rozamiento:

$$
\begin{aligned}
& \mu_{e}=\frac{F_{e}}{m_{\text {rueda }} * g}=\frac{126,8 N}{14,7 k g * 9,8 m / s^{2}}=0,88 \\
& \mu_{d}=\frac{F_{d}}{m_{\text {rueda }} * g}=\frac{10,1 N}{14,7 k g * 9,8 m / s^{2}}=0,70
\end{aligned}
$$

Las distancias de frenado se calcularon igualando la variación de la energía cinética al trabajo de la fuerza de rozamiento entre las ruedas y el piso (ecuación 5).

$$
\Delta E_{c}=W_{F r}
$$

De donde resulta la distancia a través de la ecuación (6):

$$
d=\frac{v_{i}^{2}}{2 \mu g}
$$

$\left(v_{i}\right.$ corresponde a la velocidad inicial del auto, ges la aceleración de la gravedad)

Al operar con la ecuación (5) se tomó como fuerza normal aplicada a las ruedas el propio peso del autd 6 Conceptualmente se consideró además el peso del conductor, aunque puede verse en (6), que con esto el resultado no se ve modificado. Finalmente, las distancias mínimas calculadas a partir de fueron de $45 \mathrm{~m}$ y $56 \mathrm{~m}$ para frenado sin deslizamiento y para ruedas bloqueadas respectivamente.

\footnotetext{
6 Modelo válido debido a que las cuatro ruedas frenan. La validez se sigue cumpliendo para el caso real en el que la normal correspondiente al tren delantero es mayor en condición de frenado que en el caso de vehículo inmóvil. Lo contrario ocurre en el trasero. En cualquier ocasión la suma de las normales sobre las cuatro ruedas sigue siendo igual al peso del auto (en suelo horizontal).
} 


\subsection{Actividad 4}

¿Puede despreciarse la energía cinética de rotación de las ruedas en el problema anterior?

En la actividad 3 consideraron el trabajo que hace la fuerza de rozamiento durante el frenado. Pero no deberíamos olvidar que las ruedas giran mientras el auto se desplaza, lo que agrega además energía cinética de rotación. Y durante el frenado "normal" hay que detener dicho giro, lo que demanda más trabajo alargando la distancia de frenado. Es probable que esa diferencia pueda despreciarse, pero ¿estamos seguros? ¿Qué porcentaje adicional de energía representa?

Propuesta: midiendo experimentalmente el momento de inercia de una rueda.

Se propuso el cálculo de la energía acumulada en la rotación de las cuatro ruedas durante la marcha a 100 $\mathrm{km} / \mathrm{h}$ para compararla con la energía obtenida en el problema anterior. Se recurrió a la determinación del momento de inercia $I$ de una rueda haciéndola girar (con el auto levantado, como se ve en la Figura 7) a través de una polea solidaria a la misma (llanta de bicicleta) en la cual se enrolló una cuerda. Ésta, colgada de una roldana, sostuvo en su extremo libre una masa $m(4,2 \mathrm{~kg})$.

El montaje del dispositivo se representa en la Figura 8. Al dejar libre al sistema, se obtuvo la aceleración lineal $a_{m}$ de la masampor medio de una filmación y el posterior uso de los fotogramas identificando las posiciones de dicha masa con un patrón de referencia (una regla vertical) para los instantes correspondientes 7 .

Luego, medido el radio $r_{p}$ de la polea, se logró calcular la aceleración angular de la rueda y el torque aplicado.

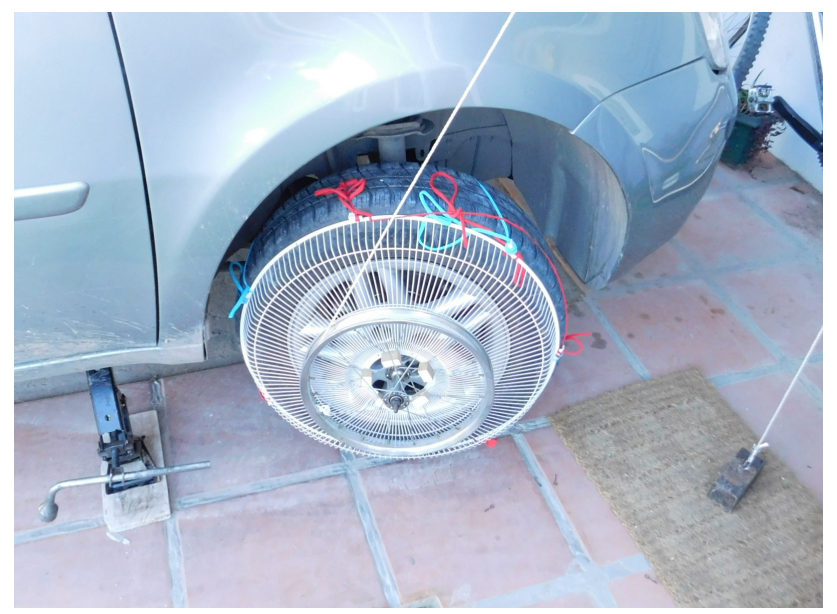

Figura 7: Rueda delantera elevada. Puede observarse la polea adosada y la cuerda con la masa en su extremo para la determinación del momento de inercia (debe hacerse con la caja de cambios en punto muerto). Debajo del tren delantero se ubicó un taco sobre el cual el vehículo apoya, además de hacerlo sobre el crique. Para mayor seguridad se aplicó el freno de mano (opera sobre ruedas traseras).

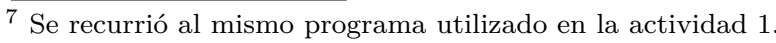

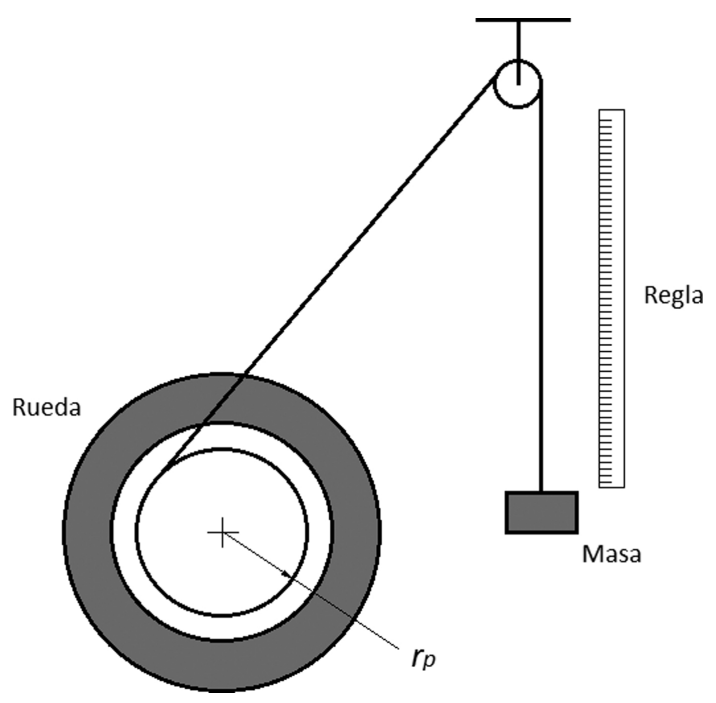

Figura 8: Esquema del montaje utilizado para la determinación del momento de inercia de una rueda.

Por otro lado, el torque puede expresarse como lo indica la ecuación (7):

$$
\tau=I * \alpha
$$

Donde $I$ es el momento de inercia y $\alpha$ la aceleración angular de la rueda ocasionada por la tensión $T$ de la cuerda. Si para un instante dado consideramos aplicada la tensión sobre un punto en la periferia de la polea, se obtiene la ecuación (8):

$$
\vec{\tau}=\vec{r}_{p} \times \vec{T}
$$

Donde se tiene un ángulo recto entre $r_{p} \mathrm{y} T$. Luego, igualando (7) y la obtenida de 8 y considerando la aceleración de la masa suspendida $a_{m}$ (ecuación 9):

$$
\vec{a}_{m}=\vec{\alpha} \times \vec{r}_{p}
$$

Resulta, aplicando la segunda ley de Newton al sistema (asumiendo condiciones "ideales" en la cuerda):

$$
\frac{I \cdot a_{m}}{r_{p}^{2}}=m g-m a_{m}
$$

Finalmente, ordenando se obtiene el momento de inercia (ecuación 10):

$$
\begin{array}{r}
I=r_{p}^{2} m\left(\frac{g}{a_{m}}-1\right)=(0,15 m)^{2} \\
\times 4,2 k g\left(\frac{9,8 m / s^{2}}{0,82 m / s^{2}}-1\right) \approx 1,034 \mathrm{kgm}^{2}
\end{array}
$$

Se obtuvo así el momento de inercia de una rueda $(1,034$ $\mathrm{kgm}^{2}$ ) con lo que finalmente se pudo calcular la energía cinética de rotación $E_{C R}$ de la misma (ecuación 11 ).

$$
\begin{aligned}
E_{C R}=\frac{1}{2} I & \left(\frac{v}{r_{r}}\right)^{2}=\frac{1}{2} * 1,034 \mathrm{kgm}^{2} \\
& \times\left(\frac{27,7 \mathrm{~m} / \mathrm{s}}{0,295 \mathrm{~m}}\right)^{2} \approx 4370 \mathrm{~J}
\end{aligned}
$$


(Aquí $r_{r}$ corresponde al radio de la rueda y $v$ la velocidad del vehículo antes de frenar)

Asumiendo el mismo valor para las cuatro ruedas 8 , el resultado obtenido se encontraba en el orden de un $4 \%$ de la energía cinética correspondiente al auto tomando en consideración únicamente su velocidad lineal $(17,5 \mathrm{~kJ}$ y $478 \mathrm{~kJ}$ respectivamente).

\subsection{Actividad 5}

Determinación de la fuerza neta que debe vencer el auto (el motor) durante su avance. ¿Será constante esa fuerza? ¿Cuál es su origen? ¿Dependerá de la velocidad?

Se sugiere considerar una fuerza media para diferentes $\Delta v$. Tengan en cuenta el uso del velocímetro.

Con el vehículo en una calzada solitaria (convenientemente elegida para no ocasionar riesgos), se lo puso en movimiento alcanzando $80 \mathrm{~km} / \mathrm{h}$ y luego se lo dejó sin tracción (en "punto muerto") de modo que vaya perdiendo velocidad sin forzar el detenimiento. Este proceso se realizó filmando el velocímetro. Se entiende que, al no conservar un valor constante de velocidad, el vehículo es lentamente detenido por aquellas fuerzas que en definitiva debe vencer el motor. Para la determinación de la fuerza neta se calcularon aceleraciones medias a partir de pequeños intervalos $\Delta v$ y $\Delta t$ de modo similar a lo realizado en la actividad 1 (ecuación 12).

$$
F_{\text {media }}=m_{(\text {auto+ocupantes })} * \frac{\Delta v}{\Delta t}
$$

Se muestra en la Figura 9 el gráfico de la velocidad en función del tiempo. Se presentan en la tabla 1 algunos valores de la fuerza media para diferentes intervalos, calculados a partir de los datos con planilla de cálculo. Los valores obtenidos permitieron construir las respuestas correspondientes a la consigna.

\subsection{Actividad 6}

¿Podría el motor lograr el levantamiento de la masa en las condiciones que muestra la figura? Considerar para

Tabla 1: Valores de la fuerza media para diferentes intervalos de velocidad calculados a partir de los datos obtenidos con el velocímetro.

\begin{tabular}{|l|l|}
\hline Intervalo de velocidad $[\mathrm{km} / \mathrm{h}]$ & Fuerza media $[\mathrm{N}]$ \\
\hline $80-70$ & 611,3 \\
$70-60$ & 516,1 \\
$60-50$ & 451,5 \\
$50-40$ & 386,1 \\
$40-30$ & 333,5 \\
$30-20$ & 301,2 \\
$20-10$ & 275,8 \\
$10-0$ & 252,5 \\
\hline
\end{tabular}

8 Aunque las cuatro ruedas sean esencialmente idénticas, montadas en el vehículo no consignan el mismo momento de inercia, principalmente porque las motrices (delanteras en nuestro caso) movilizan en su giro parte del mecanismo de transmisión. En la experiencia se despreciaron los rozamientos.

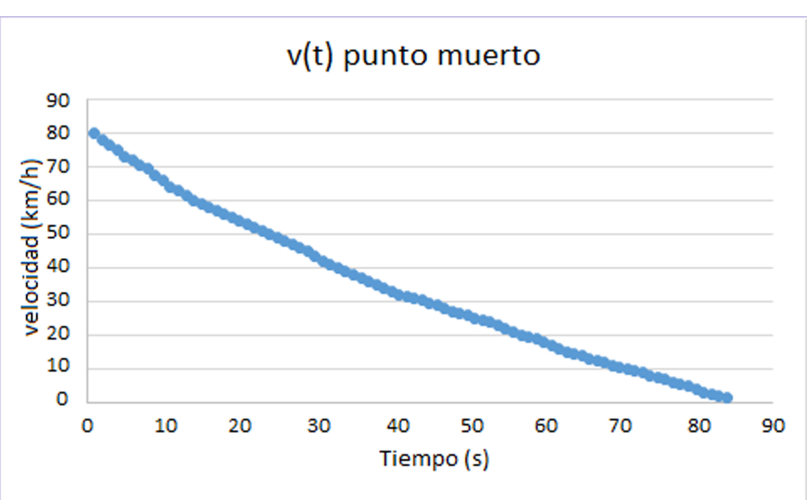

Figura 9: Gráfico de velocidad en función del tiempo con el vehículo en punto muerto. Datos obtenidos con la filmación del velocímetro. La masa considerada en el cálculo es la del vehículo y la de sus ocupantes.

el cálculo el dato del torque del motor ¿En qué marcha sugerirían hacerlo? Si puede levantarlo, ¿qué valor de aceleración máxima permitiría obtener? Despreciar las fuerzas no conservativas que disminuyen la tracción efectiva.

Para resolver el problema (esquematizado en la figura 10) se consideró la relación de transmisión de diferentes marchas, el diámetro de las ruedas y el máximo torque de motor según indica el fabricante (ecuación 13). El torque en las ruedas motrices $\tau_{r}$ será tanto mayor al del motor $\tau_{m}$ según lo establezca la relación total de velocidad de caja y de diferencial; para el caso de la $1^{\circ}$ marcha resulta de multiplicar ambas reducciones $(R C$ y $R D)$, lo que da un valor de 18,8 para esa relación (valor ya utilizado en la actividad 2 ).

$$
\tau_{r}=R C * R D * \tau_{m}=18,8 * 121,6 N m \approx 2286 N m
$$

Luego, la aceleración del auto que podría proporcionar el motor para estas condiciones (ideales), surge de aplicar la $2^{\circ}$ ley de Newton al sistema de dos ecuaciones planteadas.

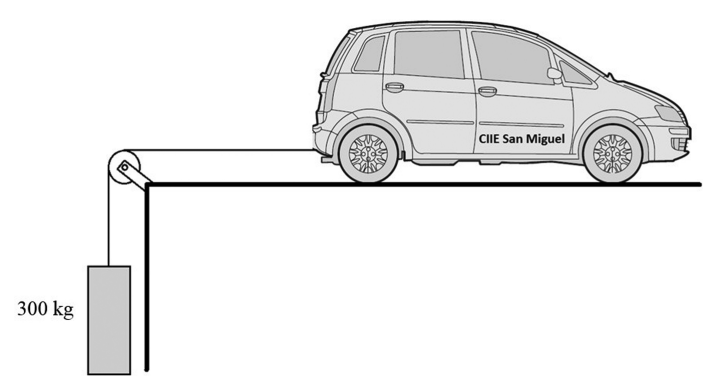

Figura 10: Esquema presentado a los docentes en la actividad 6 (imagen adaptada del manual de usuario del vehículo). 
Resolviendo resulta la ecuación 14:

$$
\begin{aligned}
a & =\frac{R C * R D * \tau_{m} *\left(r_{r}\right)^{-1}-m_{\text {bloque }} * g}{m_{\text {bloque }}+m_{\text {auto }+ \text { conductor }}} \\
& =\frac{2286,2 N m *(0,295 \mathrm{~m})^{-1}-4,2 \mathrm{~kg} * 9,8 \mathrm{~m} / \mathrm{s}^{2}}{300 \mathrm{~kg}+1250 \mathrm{~kg}} \\
& \approx 4,9 \mathrm{~m} / \mathrm{s}^{2}
\end{aligned}
$$

Es interesante resaltar que los resultados mostraron que la máxima aceleración (en $1^{\text {ra }}$ velocidad) está limitada por la adherencia de las ruedas motrices (sobre pavimento) antes que por las prestaciones del motor (las ruedas no podrían aplicar sobre el piso la fuerza que resulta del torque calculado para las mismas). Con este descubrimiento, para el cálculo de la aceleración se consideró el máximo valor de la fuerza de rozamiento estático de las ruedas que predice el modelo. Se utilizó el valor del $\mu_{\mathrm{e}}$ calculado en la actividad 3 , y para obtener la fuerza normal en las ruedas motrices se hizo uso de la balanza hogareña y una tabla de madera a modo de palanca de tercer género (Figura 11).

Este método permitió medir una fuerza superior a los $3000 \mathrm{~N}$ con una pequeña balanza limitada a $120 \mathrm{~kg}$ (Figuras 12 y 13).

Según los cálculos anteriores, el bloque imaginado podría ser levantado entonces con una aceleración instantánea de $1,9 \mathrm{~m} / \mathrm{s}^{2}$ (valor teórico correspondiente únicamente para el caso en que el motor se encuentre en su máximo par).

Se recurrió a animaciones para la visualización de los elementos (básicos) de la transmisión de un vehículo y para discutir a partir de la misma los efectos de un tren de engranajes, en términos de la Físicd 9 Esto es, la conversión de par como resultado de la relación del

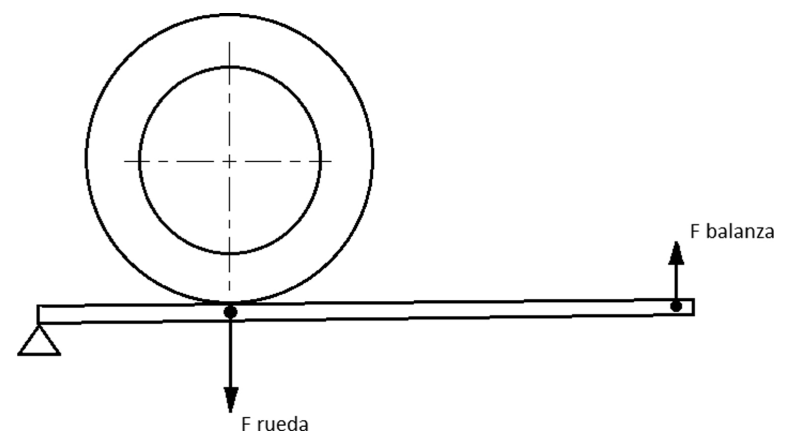

Figura 11: Esquema del dispositivo para determinar la normal en las ruedas motrices. La suma de los momentos es cero en el sistema.

\footnotetext{
9 Se eligieron para esto los videos disponibles en las siguientes direcciones (en dicho orden): https://www.youtube.com/watch?v=XfGE8shOkXc Consultado el 30 de enero de 2017, https://youtu.be/oA4ZBmMZfrc Consultado el 30 de enero de 2017, https://youtu.be/tqK0RHDM8PM Consultado el 30 de enero de 2017 .
}

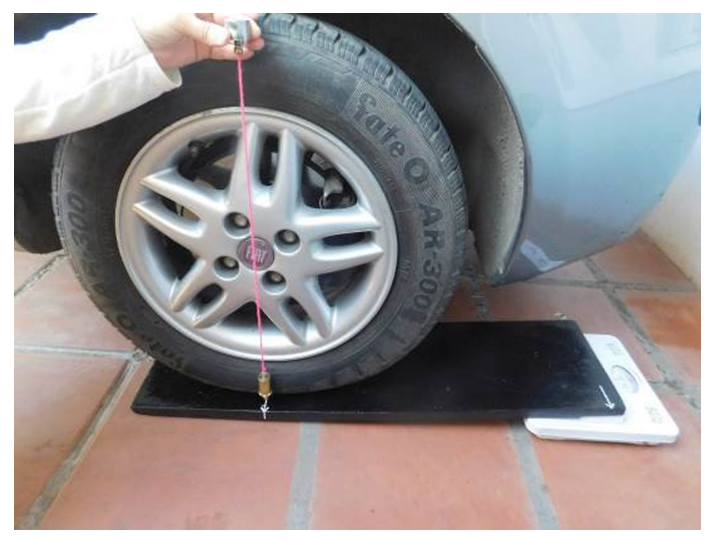

Figura 12: Montaje con tabla y balanza para determinar la normal aplicada a la rueda. En la imagen se aprecia la determinación del "punto" de apoyo con la ayuda de una plomada. Se colocó un listón de madera centrado en la balanza bajo la tabla (donde se ubica la flecha dibujada).

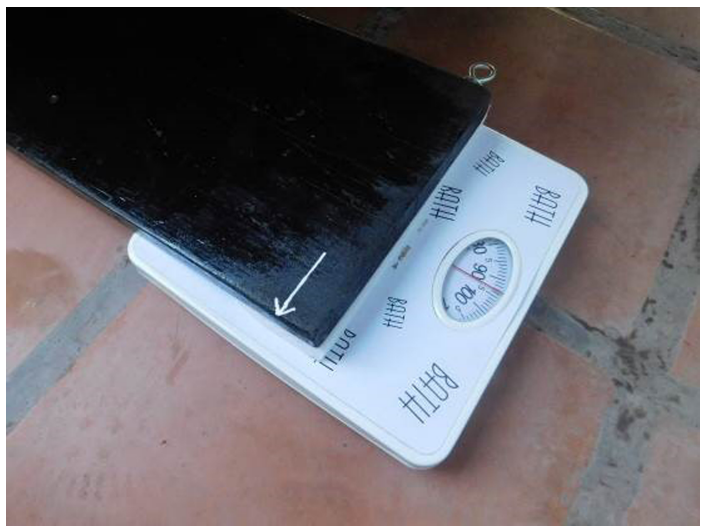

Figura 13: Lectura en la balanza como consecuencia de la acción de la rueda sobre la tabla. Se recomienda desplazar al vehículo sobre la tabla girando sus ruedas manualmente con lo que se tendrá mayor control. Si se excede la posición adecuada podría deteriorarse la balanza.

número de dientes, la ventaja del uso de engranajes helicoidales $\sqrt{10}$ entre otros.

\subsection{Actividad 7}

Sería posible tener una aproximación al rendimiento del motor en alguna situación particular de funcionamiento. Entendiendo que es una máquina térmica que transforma parte de la energía del combustible en trabajo mecánico, se propone el cálculo de la potencia suministrada por el combustible para un valor especificado de potencia entregada por el motor.

Contamos con el dato de la potencia máxima y la velocidad de rotación del motor a la cual la entrega (60,4

10 Tienen la interesante propiedad física de poder transmitir mayor potencia debido a que el contacto entre dientes de dos engranajes vinculados se produce de a pares, es decir simultáneamente dos dientes de un engranaje con dos del otro. Como ventaja adicional, resultan más silenciosos, aspecto importante en un vehículo. 
kW a 5500 RPM). Esto nos permitirá encontrar un valor para el rendimiento en esas condiciones.

Datos del combustible (nafta 95 octanos):

Relación de la mezcla combustible: $14,8 \mathrm{~g}$ de aire por $\mathrm{g}$ de nafta

Densidad: $0,740 \mathrm{~kg} / \mathrm{l}$

Poder calorífico: $10350 \mathrm{kcal} / \mathrm{l}$

Esta actividad, "más de salón", se pudo resolver con los datos anteriores y planteando un modelo de funcionamiento con algunas simplificaciones, dado que las condiciones reales de funcionamiento de un motor resultan ser más complejas.

A partir del dato de las 5500 RPM (donde entrega la máxima potencia) y de la cilindrada (volumen total de los cilindros del motor), se calculó el volumen teórico aspirado (VTA) por unidad de tiempo a través de la ecuación (15):

$$
V T A=\frac{R P M * \text { cilindrada }}{2 * 60 s / \operatorname{mín}}=62,7 l / s
$$

Acá es importante señalar que, tratándose de un motor de cuatro tiempos, la aspiración correspondiente a la cilindrada $(1,368 l)$ se completa en dos vueltas.

La relación estequiométrica aire- nafta ${ }^{11}$ permitió establecer la masa de combustible ingresada por volumen de mezcla (aire- nafta) aspirado $(M C A)$. Luego se consideró además un valor de 0,8 para el llenado volumétrico $(L L V)^{12}$ Con el dato del poder calorífico $(P C)$, finalmente se calculó la potencia asociada a la cantidad de combustible que ingresa. Todo esto puede verse en una única expresión dada por la ecuación 16 :

$$
\begin{gathered}
P=V T A * M C A * L L V * P C \\
P=\frac{62,7 l * 0,0877\left(g_{\text {nafta }} / l_{\text {mezcla }}\right) * 0,8 * 10350 \mathrm{cal} / \mathrm{g}}{\mathrm{s}} \\
\approx 45530 \mathrm{kcal} / \mathrm{s}
\end{gathered}
$$

Este último resultado, expresado en Watt, fue comparado con el dato de la potencia entregada por el motor (190 $\mathrm{kW}$ y 60,4 kW respectivamente) A través del cociente entre ambos valores se obtuvo finalmente el rendimiento (en el orden del $32 \%$ para las condiciones establecidas).

Para esta actividad resultó conveniente el uso de una animación (editada) para facilitar el entendimiento del funcionamiento interno del motor 13

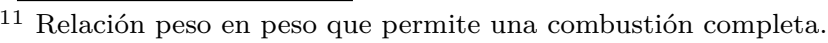

12 En un motor de aspiración natural (no sobrealimentado), y particularmente en alta velocidad de giro, la presión dentro de los cilindros al final de la admisión suele ser menor a la atmosférica, lo cual hace que el número de moles ingresado sea menor al esperado. Si bien no contamos con el dato real del fabricante, utilizamos un valor típico de llenado volumétrico que representa la fracción del volumen total.

13 Se utilizó el video disponible en: www.youtube.com/watch?v $=58$ OfsvsFDtU Consultado el 30 de enero de 2017
}

\section{Evaluación de las actividades}

Algunas de las situaciones registradas en torno a las actividades implementadas se presentan a continuación. Esto puede ser de utilidad para quienes se interesen en reproducirlas.

La primera actividad indujo discusiones valiosas en relación al concepto de aceleración en fenómenos reales como es el caso del desplazamiento de un automóvil. En efecto, se tuvo el caso de algunos participantes que consideraban la posibilidad, a priori, de una modelización a través de un "MRUV", quizá inducido por los clásicos ejercicios de nivel medio donde se aplica a diferentes casos ideales, que incluyen hipotéticos automóviles. Era esperable que la aceleración no fuera constante, aunque en principio no resultaría sencillo evaluar en qué medida variaría. Se tienen, en este sentido, dos factores antagónicos. Por un lado, el aumento de la potencia del motor como función de su velocidad angular hasta que aquella alcanza un valor máximo y, por el otro, el crecimiento de las fuerzas no conservativas al aumentar la velocidad del vehículo, las que tienen como causa principal a la fricción con el aire y a la deformación de los neumáticos. A juzgar por los resultados, parecería que el segundo factor se impuso levemente. Sin embargo, esta conclusión la hemos puesto en cuestión debido a la relativa poca precisión del método utilizado para la toma de datos. Si bien puede resultar sencillo lograr una filmación de calidad del velocímetro, éste suele ser un instrumento no demasiado preciso debido al uso para el cual es concebido. Por otra parte, los resultados que obtuvimos con el péndulo no avalan de modo indiscutible el resultado debido a lo poco estable de su comportamiento ya que se producen oscilaciones indeseables. Cabe aclarar que en la bibliografía confirman el decrecimiento de la aceleración con la velocidad de un vehículo, aunque en ocasiones sin la debida aclaración si la causa radica en las diferentes relaciones de marcha de la caja de velocidades (puede verse esto en [8], pág. 57).

La actividad 2 resultó adecuada situarla antes de las siguientes dentro de una "hipótesis de progresión" en términos de García [9], quien hace referencia a la construcción gradual y progresiva de una determinada idea como a un conjunto de contenidos conectados entre sí en una trama. En ese sentido, la problematización con conceptos propios del movimiento circular se retomó y se profundizó en las actividades 4 y 6 . Particularmente se valoró la utilidad práctica de la balanza hogareña para otras disposiciones posibles que exceden a su uso tradicional. Cabe señalar que dichos dispositivos tienen la ventaja de contar con una escala considerablemente amplia. Se recuerda aquí la conveniencia de permanecer un conductor dentro del vehículo durante el empuje manual por una eventual necesidad de frenarlo (con el motor en marcha).

Las actividades 3 y 4, relacionadas con el frenado de un vehículo, fueron especialmente apreciadas por ser 
integradoras de varios contenidos de Física escolar dentro de una situación problemática. Las mediciones fueron relativamente sencillas de realizar y, en opinión de todo el grupo, con métodos bastante confiables (y de baja incerteza). Por ejemplo, tomar la posición en función del tiempo a partir de los fotogramas en la actividad 4 permitió identificar una gran cualidad que ofrece el análisis de video y que es la "naturalidad" con la que pueden ser examinados movimientos que ocurren en un plano [10]. Por otro lado, la determinación experimental de la energía de rotación la consideramos valiosa para trabajar un tema que resulta complejo a los estudiantes; el de la rotación del sólido rígido, a pesar de las múltiples aplicaciones de este tema en la ciencia y en la vida cotidiana [11]. Las actividades 3 y 4 además indujeron la discusión sobre la situación real en la cual el tiempo de reacción del conductor aumenta la distancia calculada, la necesidad de mantener distancia prudencial, el riesgo que representa el bloqueo de las ruedas, etc. Con la actividad resultó particularmente reveladora la cuantificación de la energía disipada (y "desperdiciada") en el sistema de frenos ya que arroja valores relativamente elevados (en el orden de 0,5 MJ en nuestro ejemplo).

Con la actividad 5 se logró poner de manifiesto de modo evidente la variación permanente de la resistencia al avance con la velocidad. Esto, que en definitiva fue una confirmación de algo esperado, podría ser de valor para los docentes teniendo en cuenta que el hábito escolar en la enseñanza de la Física conlleva casi exclusivamente la consideración de fuerzas constantes (como una de las pocas excepciones consideradas se tiene la ley de Hooke). Ejemplos como el trabajado podrían ser de interés para un tratamiento cualitativo de fuerzas variables en aquel ámbito. Cabe recordar que el origen de la variación con la velocidad debemos atribuirlo principalmente al rozamiento con el aire y a la deformación dinámica de los neumáticos durante la rodadura (con lo cual depende fuertemente de la presión del aire dentro de los mismos). Del mismo modo que la actividad 1, la precisión en los resultados se liga directamente a la relativa confiabilidad que ofrece el velocímetro analógico de un vehículo para uso familiar.

La actividad 6 parte de un esquema más o menos tradicional en la enseñanza de la dinámica, aunque con el posible atractivo de la toma de datos con el vehículo además del uso de las especificaciones reales de su planta motriz. Aunque, en relación a ésta y como ya se anticipó, la limitación impuesta por la fuerza de rozamiento fue una cuestión relevante en la resolución. Por otro lado, dada las condiciones ideales para las cuales fue calculada la aceleración, hubo acuerdo entre los docentes en que los valores reales serían significativamente menores. Es de resaltar el significado que tuvo el concepto de "máxima aceleración"; resulta un valor teórico instantáneo que no puede conservarse ya que al ganar velocidad implica un aumento de las RPM del motor, con lo cual nuevamente decrece su torque. Se realizó además la comparación con el "torque teórico en el motor" necesario para sostener la aceleración medida en la actividad 1 y, efectivamente, arroja un valor bastante menor al valor brindado por el fabricante. Esta discrepancia la interpretamos como el resultado de la simplificación del modelo que utilizamos. Lejos de subvalorar al mismo, lo hemos considerado de gran utilidad en virtud de las discusiones generadas. El uso de una tabla como palanca para poder valerse de la balanza fue particularmente apreciado por su utilidad práctica (y pedagógica considerándola como un caso de palanca de tercer género). Vale aclarar que valiéndonos del recurso se repitió la medición en las demás ruedas con lo que se determinó con notable precisión el peso total del auto.

En relación a la actividad 7 se tuvo acuerdo en que resultó un método simple y razonable para el cálculo del rendimiento. El resultado, sin embargo, es muy dependiente del valor asignado al llenado volumétrico; allí podría tenerse la mayor incerteza ya que se tomó un valor no verificado para el motor considerado. Abrió un interesante debate en torno al concepto de rendimiento aplicado a un motor térmico muy presente en la vida cotidiana y, con los resultados, una reflexión en torno a las significativas pérdidas por degradación de la energía y sus indeseables consecuencias para el ambiente. Acordamos con Martín et. al. [12] en que contextualizar el problema de la energía en la vida cotidiana es conveniente para familiarizar a los estudiantes con sistemas reales no idealizados, estableciendo conexiones entre problemas ligados a la energía y eventos reales de la vida, propiciando una toma de conciencia sobre su consumo. Según palabras de algunos de los docentes, el concepto de rendimiento aplicado a máquinas térmicas sólo había sido abordado de manera teórica con el estudio del ciclo de Carnot, en su formación inicial.

\section{Conclusiones}

Se realizó una encuesta de opinión al final del curso donde los docentes manifestaron que las actividades generaron gran interés y permitieron un sostenido intercambio en torno a la resolución, a los alcances y limitaciones de los modelos empleados y a la precisión en las mediciones como su influencia en los resultados entre otros aspectos técnicos. Asimismo, en aquella encuesta se evaluaron las fortalezas y debilidades de la propuesta de trabajo y sobre la posibilidad de adaptar algunas de las actividades a la escuela secundaria (entendemos que ciertas actividades, particularmente las realizadas con el vehículo en movimiento en la vía pública no podrían realizarse en secundaria). Hubo consenso en el grupo en la consideración de que algunas de aquellas fortalezas se relacionaban con el montaje de dispositivos construidos con elementos sencillos, las mediciones específicas y la modelización, tanto física como matemática y que permite ser resuelta con los recursos informáticos actualmente disponibles por los docentes. 
Se asume que la problematización de contenidos en un formato de curso como el propuesto, puede enriquecer la práctica docente favoreciendo el aprendizaje de los alumnos y promoviendo formas de trabajo cercanas a las metodologías científicas en el planteo de problemas de Física escolar.

\section{Referencias}

[1] J. Fernández, N. Elórtegui y M. Pérez, Revista Electrónica de Enseñanza de las Ciencias 2, 263 (2003).

[2] V. Mellado Jiménez, Enseñanza de las Ciencias 21, 343 (2003).

[3] A. García-Carmona, Revista Electrónica de Enseñanza de las Ciencias 10(Núm. Extraordinario), 552 (2013).

[4] L. Vygotsky, Pensamiento y Lenguaje (Pléyade, Buenos Aires, 1985).

[5] R. Lara, La Cooperación en la Educación Superior: Una Metodología Didáctica para Trabajar en el Aula (PraxisUAEH, México, 1997).

[6] E. Roditi, La Résolution de Problèmes Professionnels, Une Modalitè de Formation Continue des Enseignants (Université Paris VII, Paris, 2004).

[7] E. Cyrulies (coord.) Experiencia de Trabajo Cooperativo en Formación Docente Vinculando el Frofesorado de Física y el de Educación Primaria: Exploración de las Intervenciones de los Estudiantes Aprendiendo Contenidos de Astronomía en Grupos Heterogéneos (INFOD, Buenos Aires, 2015).

[8] H. Young, y R. Freedman Física Universitaria, Vol. 1 (Pearson Education, México, 2009), $12^{\mathrm{a}}$ ed.

[9] J. García, Hacia una Teoría Alternativa Sobre los Conocimientos Escolares (Díada, Sevilla, 1998).

[10] L. Navarrete Navarrete, F. Almaguer Medina, F. Navarrete Navarrete y M. Flores Pérez, Latin American Journal of Physics Education 9, 3402 (2015).

[11] M. Fernández Dieguez, L. Rodríguez Landrove, I. Marcheco Puig, N. Jiménez Sánchez, L.Pallerols Sánchez, y M. Hechavarría Rodríguez, Latin American Journal of Physics Education 9, 1503 (2015).

[12] C. Martín, T. Prieto y M. Jiménez, Revista Eureka sobre Enseñanza y Divulgación de las Ciencias 10, 649 (2013). 